\title{
Molecular lines in the envelopes of evolved stars
}

\author{
Yong Zhang ${ }^{1}$, Sun Kwok ${ }^{1}$, and Dinh-V-Trung ${ }^{2}$ \\ ${ }^{1}$ Department of Physics, University of Hong Kong, Pokfulam Road, Hong Kong \\ email: zhangy96@hku.hk; sunkwok@hku.hk \\ ${ }^{2}$ Institute of Astronomy and Astrophysics, Academia Sinica, P.O Box 23-141, \\ Taipei 106, Taiwan \\ email: trung@asiaa.sinica.edu.tw
}

\begin{abstract}
We report a spectral line survey of the circumstellar envelopes of evolved stars at millimeter wavelengths. The data allow us to investigate the chemical processes in different physical environments and evolutionary stages. A total of more than 500 emission features (mostly rotational transitions of molecules) are detected in the survey. Our observations show that the sources in different evolutionary stages have remarkably different chemical composition. As a star evolves from AGB stage to proto-planetary nebula, the abundances of Si-bearing molecules ( $\mathrm{SiO}, \mathrm{SiCC}$, and $\mathrm{SiS}$ ) decrease, while the abundances of some long-chain molecules, such as $\mathrm{CH}_{3} \mathrm{CN}, \mathrm{C}_{4} \mathrm{H}$, and $\mathrm{HC}_{3} \mathrm{~N}$, increase. After further evolution to planetary nebula, the abundances of neutral molecules dramatically decrease, and the emission from molecular ions becomes more intense. These differences can be attributed to the changes of the role that dust, stellar winds, shock waves, and UV/X-rays from the central star play in different evolutionary stages. These results will provide significant constraints on models of circumstellar chemistry.
\end{abstract}

Keywords. Stars: AGB and post-AGB, line: identification, ISM: molecules, radio lines: ISM

\section{Introduction}

Since 1970, more than 60 molecular species, including many organic molecules, have been detected in the circumstellar envelopes of evolved stars. Asymptotic giant branch (AGB) stars, their descendant planetary nebulae (PNs), and the transition objects between the two phases, proto-planetary nebulae (PPNs), therefore represent major sites of molecular synthesis. Since the evolutionary time scales of these phases are very short $\left(10^{4}-10^{5} \mathrm{yr},<10^{3} \mathrm{yr}, 10^{3}-10^{4} \mathrm{yr}\right.$ for AGB stars, PPNs, and PNs, respectively), chemical reaction time scales are very well constrained by these time scales. The study of the changing chemical composition and molecular abundance between objects in consecutive phases of evolution provides useful information on the chemical pathways of molecular synthesis. Furthermore, these comparisons can lead us to a better understanding of the roles of dust, shock waves, and UV and X-ray radiation on the chemical processes. For this purpose, we present a molecular line survey in a sample of evolved stars.

\section{Observations}

Our sample consists of three AGB stars (IRC+10216, CRL 3068, and CIT 6), one PPN (CRL 2688), and one young PN (NGC 7027). The spectral survey was carried out between 2005 April and 2008 January using the Arizona Radio Observatory $12 \mathrm{~m}$ and $10 \mathrm{~m}$ telescopes, covering the frequency ranges from $71-161 \mathrm{GHz}$ and $218-268 \mathrm{GHz}$, respectively. The typical sensitivity is $T_{\mathrm{R}}<10 \mathrm{mK}$ at a spectral resolution of $1 \mathrm{MHz}$. The spectra are presented in Figure 1. The temperature scales at the ARO $12 \mathrm{~m}$ and $10 \mathrm{~m}$ are $T_{R}^{*}$ and $T_{A}^{*}$, respectively. The main beam brightness temperatures were derived through 

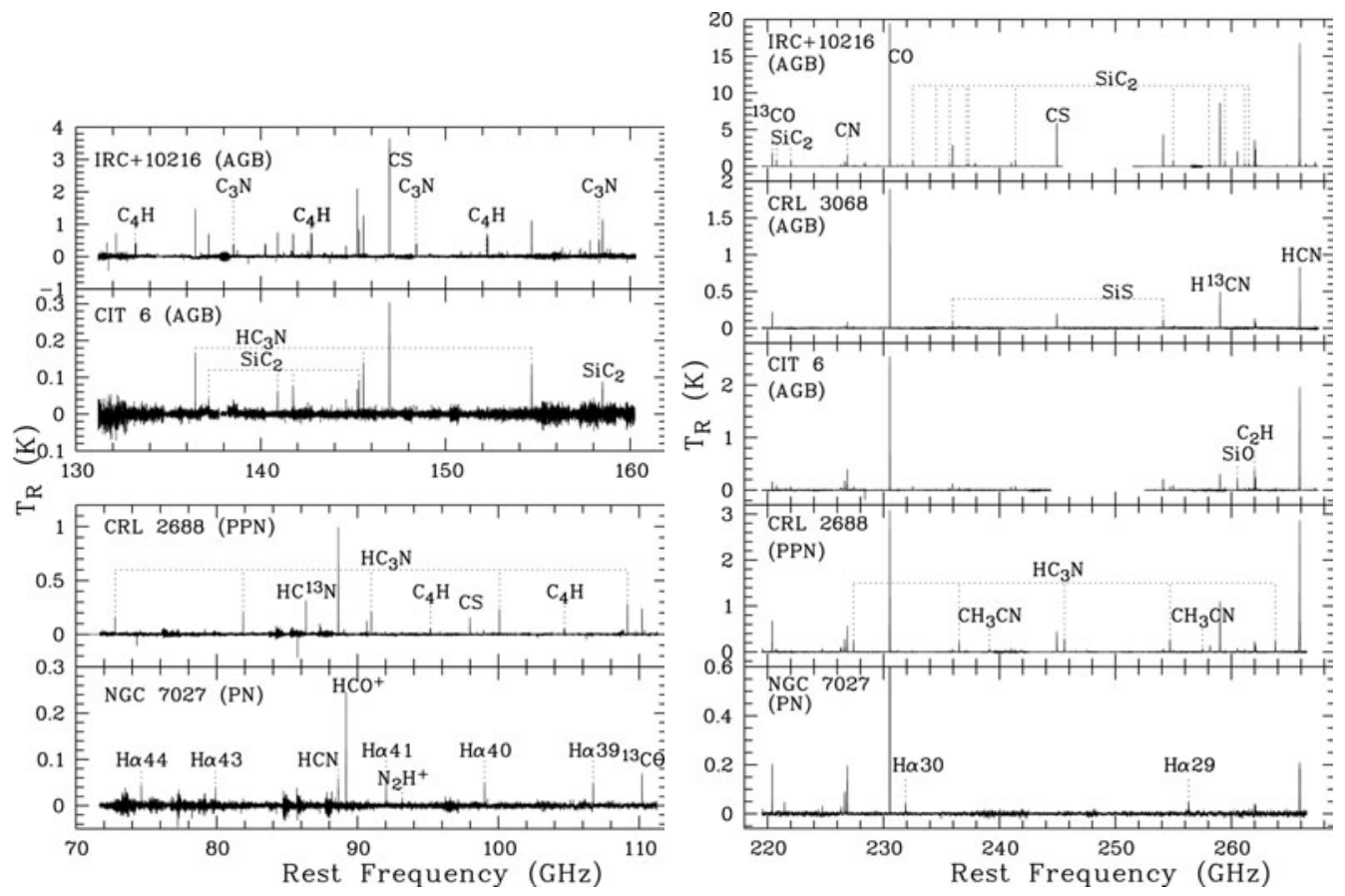

Figure 1. Left panel: The $12 \mathrm{~m}$ data; Right panel: The $10 \mathrm{~m}$ data.

$T_{R}=T_{R}^{*} / \eta_{m}^{*}$ and $T_{R}=T_{A}^{*} / \eta_{m b}$, where $\eta_{m}^{*}$ is the corrected beam efficiency and $\eta_{m b}$ the beam efficiency.

\section{Conclusions}

A systematic comparison of the molecular emission in the five objects has revealed the following spectral differences:

a) Refractory metal- and Si-bearing species, which are plentifully present in IRC+10216, show weak or no emission in the other objects. These molecules may be depleted onto dust grains with stellar evolution.

b) Compared to the other objects, CRL 2688 has stronger $\mathrm{C}_{4} \mathrm{H}, \mathrm{HC}_{3} \mathrm{~N}$ and $\mathrm{CH}_{3} \mathrm{CN}$ emission, suggesting that these species are rapidly reprocessed during the evolution from AGB to PPN.

c) Compared to other objects, CIT 6 has a large $\mathrm{CN} / \mathrm{HCN}$ intensity ratio due to photodissociation of $\mathrm{HCN}$ into $\mathrm{CN}$. However, the CN/HCN ratios in CRL 2688 and NGC 7027 are relatively low, indicating that $\mathrm{CN}$ and/or HNC may be efficiently transferred into $\mathrm{HCN}$ in hot environments.

d) Emissions from neutral molecules in NGC 7027 are fainter compared to those in other objects. This is partly due to the destruction of neutral molecules caused by strong shocks and UV radiation in the young $\mathrm{PN}$.

e) For our observations, NGC 7027 is the only source in which we have detected ionized species $\left(\mathrm{HCO}^{+}, \mathrm{HCS}^{+}\right.$, and $\left.\mathrm{N}_{2} \mathrm{H}^{+}\right)$and recombination lines. This is attributed to photoionization by the much stronger UV radiation from the central star.

Further observations are underway to expand the evolutionary coverage in order to better constrain the relation between chemical abundance and physical conditions. 